\title{
Is there added value in obtaining cervical spine MRI in the assessment of nontraumatic angiographically negative subarachnoid hemorrhage? A retrospective study and meta-analysis of the literature
}

\author{
Gelareh Sadigh, MD, ${ }^{1}$ Chad A. Holder, MD, ${ }^{1}$ Jeffrey M. Switchenko, PhD, ${ }^{2}$ \\ Seena Dehkharghani, MD, ${ }^{3}$ and Jason W. Allen, MD, PhD ${ }^{1,4}$ \\ 'Department of Radiology and Imaging Sciences, Emory University School of Medicine, Atlanta, Georgia; 2Department of \\ Biostatistics and Bioinformatics, Rollins School of Public Health, Atlanta, Georgia; ${ }^{3}$ Department of Radiology, NYU School of \\ Medicine, New York, New York; and ``Department of Neurology, Emory University School of Medicine, Atlanta, Georgia
}

OBJECTIVE Diagnostic algorithms for nontraumatic angiographically negative subarachnoid hemorrhage (AN-SAH) vary, and the optimal method remains subject to debate. This study assessed the added value of cervical spine MRI in identifying a cause for nontraumatic AN-SAH.

METHODS Consecutive patients 18 years of age or older who presented with nontraumatic SAH between February 1 , 2009, and October 31, 2014, with negative cerebrovascular catheter angiography and subsequent cervical MRI were studied. Patients with intraparenchymal, subdural, or epidural hemorrhage; recent trauma; or known vascular malformations were excluded. All cervical MR images were reviewed by two blinded neuroradiologists. The diagnostic yield of cervical MRI was calculated. A literature review was conducted to identify studies reporting the diagnostic yield of cervical MRI in patients with AN-SAH. The weighted pooled estimate of diagnostic yield of cervical MRI was calculated.

RESULTS For all 240 patients (mean age 53 years, $48 \%$ male), catheter angiography was performed within 4 days after admission (median 12 hours, interquartile range [IQR] 10 hours). Cervical MRI was performed within 19 days of admission (median 24 hours, IQR 10 hours). In a single patient, cervical MRI identified a source for SAH (cervical vascular malformation). Meta-analysis of 7 studies comprising 538 patients with AN-SAH produced a pooled estimate of $1.3 \%$ (95\% confidence interval $0.5 \%-2.5 \%)$ for diagnostic yield of cervical MRI. No statistically significant between-study heterogeneity or publication bias was identified.

CONCLUSIONS Cervical MRI following AN-SAH, in the absence of findings to suggest spinal etiology, has a very low diagnostic yield and is not routinely necessary.

https://thejns.org/doi/abs/10.3171/2017.4.JNS163114

KEY WORDS added value; cervical spine MRI; nontraumatic angiographically negative subarachnoid hemorrhage; meta-analysis; vascular disorders

$\mathrm{T}$ HE estimated incidence of nontraumatic subarachnoid hemorrhage (SAH) is 9 per 100,000 personyears, ${ }^{5}$ and an underlying cause, such as a ruptured aneurysm, is identified in the majority of the cases. ${ }^{2}$ In approximately $15 \%-20 \%$ of nontraumatic $\mathrm{SAH}$, angiographic evaluation fails to identify a source. ${ }^{1,10}$

The diagnostic algorithm for patients with nontraumatic angiographically negative SAH (AN-SAH) varies between institutions, and the optimal algorithm remains subject to debate. ${ }^{10,19}$ Patients with a perimesencephalic pattern of $\mathrm{SAH}$ on noncontrast head CT at presentation usually have a more benign clinical course, and some studies suggest that a combination of negative CT angiography (CTA) of the head and neck and negative digital subtraction angiography (DSA) is sufficient to exclude vascular etiologies in this population. ${ }^{25}$ However, in patients with nonperimesencephalic, nonaneurysmal patterns of SAH, the optimal diagnostic algorithm remains inconclusive. Anecdotal evi-

ABBREVIATIONS AN = angiographically negative; $\mathrm{C}-\mathrm{MRI}=$ cervical $\mathrm{MRI} ; \mathrm{Cl}=$ confidence interval; $\mathrm{CTA}=\mathrm{CT}$ angiography; $\mathrm{DSA}=$ digital subtraction angiography; EMR = electronic medical record; FA = flip angle; IQR = interquartile range; QUADAS = Quality Assessment of Diagnostic Accuracy Studies; SAH = subarachnoid hemorrhage. SUBMITTED December 11, 2016. ACCEPTED April 28, 2017.

INCLUDE WHEN CITING Published online October 13, 2017; DOI: 10.3171/2017.4.JNS163114. 
dence of underlying vascular etiologies in patients with a perimesencephalic pattern of SAH is present in the literature, ${ }^{24,26}$ and this remains a diagnosis of exclusion.

In many centers, including our institution, patients with nontraumatic AN-SAH (negative DSA and head and neck CTA) also undergo evaluation with concurrent MRI of the brain and MRI of the cervical spine, regardless of whether they have a specific clinical or neurological finding suggestive of a spinal etiology.$^{16}$ Limited previous studies have reported a diagnostic yield of $0 \%-4 \%$ for cervical MRI (c-MRI) in this patient population, although methodologies between studies have differed and individual studies have only included a limited number of patients. . $^{10,15,16,19,22,25}$ Based on our experience, we hypothesize that c-MRI has little to no diagnostic yield in the evaluation of this patient population.

The purpose of the current study was to assess the added value of c-MRI in identifying a cause for AN-SAH in patients without clinical or neurological findings suggestive of a spinal origin using a retrospective study design. This was supplemented by a meta-analysis of the literature to assess the aggregate diagnostic yield of c-MRI in the diagnostic algorithm of patients with AN-SAH.

\section{Methods}

Emory University institutional review board approval and a waiver of informed consent were obtained for this retrospective review. The study was compliant with the Health Insurance Portability and Accountability Act.

\section{Retrospective Study}

\section{Study Population}

The Radiology Information System database was searched for consecutive patients 18 years of age or older, with acute nontraumatic intracranial $\mathrm{SAH}$, who were diagnosed based on noncontrast head CT or xanthochromia on lumbar puncture and evaluated between February 1, 2009, and October 31, 2014, at Emory University Hospital and Emory University Hospital Midtown. Inclusion criteria included patients with c-MRI whose initial cerebrovascular catheter angiography failed to reveal a cause for the SAH (identified as AN-SAH patients). Patients with acute intraparenchymal, subdural, or epidural hemorrhage; cerebral contusion; recent history of trauma or spine surgery; and with an already known origin for SAH diagnosed on CTA or DSA from prior admissions were excluded.

\section{Cervical Spine MRI Technique}

All but 4 patients (2\%) included in this study underwent c-MRI with and without intravenous contrast administration. Of the 4 patients who underwent c-MRI without intravenous contrast, 1 underwent MR angiography of the neck with intravenous contrast, which allowed postcontrast assessment of the cervical spine.

MRI was performed on either a 3.0-T (TIM Trio, Siemens Healthcare) or 1.5-T (Avanto, Siemens Healthcare; or Intera, Phillips) using a head-neck array coil for cervical spine imaging. A standard dose $(0.1 \mathrm{mmol} / \mathrm{kg})$ of gadobenate dimeglumine (MultiHance, Bracco Diagnostics) was injected at $2-3 \mathrm{ml} / \mathrm{sec}$. The standardized
MRI protocol obtained included a precontrast sagittal T1-weighted (TE $10 \mathrm{msec}$, TR $400 \mathrm{msec}$, flip angle (FA) $90^{\circ}$, slice thickness $3 \mathrm{~mm}$ ), sagittal T2-weighted (TE 90 msec, TR $2500 \mathrm{msec}$, FA $90^{\circ}$, slice thickness $3 \mathrm{~mm}$ ), axial T2-weighted (TE $90 \mathrm{msec}$, TR $5300 \mathrm{msec}$, FA 90², slice thickness $3 \mathrm{~mm}$ ), and/or postcontrast sagittal T1-weighted (TE $10 \mathrm{msec}$, TR $400 \mathrm{msec}$, FA 90 $90^{\circ}$, slice thickness $3 \mathrm{~mm}$ ) and axial T1-weighted (TE $10 \mathrm{msec}$, TR $1600 \mathrm{msec}$, FA $90^{\circ}$, slice thickness $3 \mathrm{~mm}$ ) images of the cervical spine.

\section{Medical Record Review}

Data including demographics (age, sex, and race), method of diagnosis of SAH (lumbar puncture vs noncontrast head CT), time interval between onset of symptoms (documented in history of present illness in the electronic medical record [EMR]) and diagnosis of SAH, time interval between admission and catheter angiography, time interval between admission and c-MRI, and the Hunt and Hess classification grade $^{14}$ at presentation were obtained from the EMR.

\section{Imaging Review}

The first noncontrast head CT scan that was obtained within 24 hours of admission (and prior to a brain MR image in our study population) was reviewed, and the pattern of SAH was assessed. A Fisher grade ${ }^{8}$ was also calculated for each patient on this initial head CT scan. If a head CT scan was not obtained until after the brain MR image was obtained, or if no head CT image was available, neither the pattern of hemorrhage nor the Fisher grade was determined. It was decided to exclude those head CT images obtained later in the admission because these would no longer adequately represent the pattern of SAH at onset due to redistribution.

Cervical spine MR images were reviewed by two neuroradiologists with certificates of additional qualification and 5-7 years postsubspecialty training experience (J.W.A. and C.A.H.), who were blinded to the c-MRI result as well as prior imaging, but were aware that the patient had SAH. For each patient, the cervical spine MR image was evaluated for potential pathology that might explain the patient's $\mathrm{SAH}$, including vascular malformations and intradural masses. Significant incidental findings on these studies were also recorded at the discretion of the reviewers.

\section{Meta-Analysis}

A systematic review was conducted using meta-analytical approaches outlined in the Cochrane Handbook for Systematic Reviewers ${ }^{12}$ and reported according to the Preferred Reporting Items for Systematic Reviews and MetaAnalyses (PRISMA) criteria. ${ }^{18}$

\section{Literature Search Strategy}

A literature search was conducted to identify relevant articles in PubMed (from January 1, 1966, through September 23, 2015), EMBASE (from January 1, 1974, through September 23, 2015), and the Cochrane Library databases (from January 1, 2000, through September 23, 2015). The following combination of medical subject headings or relevant key words were used for the literature 
search: [subarachnoid hemorrhage or SAH] and [angio or angiography] and [negative] and [cervical or cervical spine] and [magnetic resonance imaging or MRI]. This search strategy was combined with a manual search of references listed in identified articles.

Studies reporting the diagnostic yield of c-MRI in adult patients with nontraumatic AN-SAH were included. Surveys, review articles, case reports, studies not published in the English language, not in full text in peer-reviewed journals, or not assessing diagnostic yield of c-MRI (e.g., only assessing diagnostic yield of brain MRI or craniocervical junction) were excluded.

\section{Data Extraction and Quality Assessment}

The quality of eligible studies was evaluated using the Quality Assessment of Diagnostic Accuracy Studies (QUADAS-2) tool, ${ }^{23}$ assessing quality in 4 main categories, including patient selection, index test, reference standard, and flow and timing. Each category had several subcategory quality items that were judged to be fulfilled, not fulfilled, or not clear from the paper whether the item was fulfilled. Furthermore, the full text of each article was abstracted according to a standardized form for studies meeting the criteria. The primary outcome was recorded as the diagnostic yield of c-MRI in patients with nontraumatic AN-SAH.

\section{Statistical Analysis}

For the retrospective portion of our study, categorical variables were reported as frequency and percentage. $\mathrm{Nu}-$ merical variables were presented as mean and standard deviation (SD) or median and interquartile range (IQR) if they did not follow a normal variation. Diagnostic yield for c-MRI was calculated as a proportion of patients with an identifiable origin for SAH determined on the imaging review. The diagnostic yield of c-MRI has been reported between $0 \%$ and $4 \%$ in the current literature..$^{10,15,16,19,22,25}$ Assuming the diagnostic yield of c-MRI would be $2 \%$ in patients with AN-SAH and a diagnostic yield of 5\% is considered clinically significant, with a Type I error rate of 0.05 , and power of $80 \%$, the estimated sample size was 233 patients.

For the meta-analysis portion of our study, the proportion of patients with AN-SAH who had a positive cervical spine MR image was reported for each study, along with 95\% exact confidence intervals (CIs). To estimate the overall proportion, a Freeman-Tukey double arcsine transformation was applied and a weighted summary proportion was calculated using both fixed- and random-effects models, ${ }^{6,18}$ and demonstrated using a forest plot. For fixed-effects and random-effects model calculations, studies were weighted based on their inverse variance. In a fixed-effects model, total variance was measured only on the basis of within-study variance. In a random-effects model, total variance was measured on the basis of both within-study and between-study variance. As between-study variance becomes larger, studies become more equally weighted.

Between-study heterogeneity was assessed using the Q chi-square statistic, where a statistically significant test statistic would indicate evidence of variability across effect sizes exceeding what would be expected based on sampling error. ${ }^{13}$ The percentage of variation due to heterogeneity, $\mathrm{I}^{2}$, was estimated. Publication bias was assessed using a funnel plot of the study standard errors versus the estimated study proportions. ${ }^{20}$

The analyses were performed using STATA (version 10, Stata Corp.) for the retrospective portion, and R statistical package "meta" for the meta-analysis; $p$ values $<0.05$ were considered statistically significant.

\section{Results}

\section{Retrospective Study}

Study Population

Electronic medical records for 262 patients with nontraumatic SAH who underwent c-MRI during the same admission were reviewed (Fig. 1). After excluding 22 patients with aneurysms $(\mathrm{n}=13)$, intraparenchymal or subdural hemorrhage $(n=8)$, or recent thoracic spine surgery $(\mathrm{n}=1), 240$ patients with AN-SAH (116 men and

\section{2 patients with SAH and cervical spine MRI}

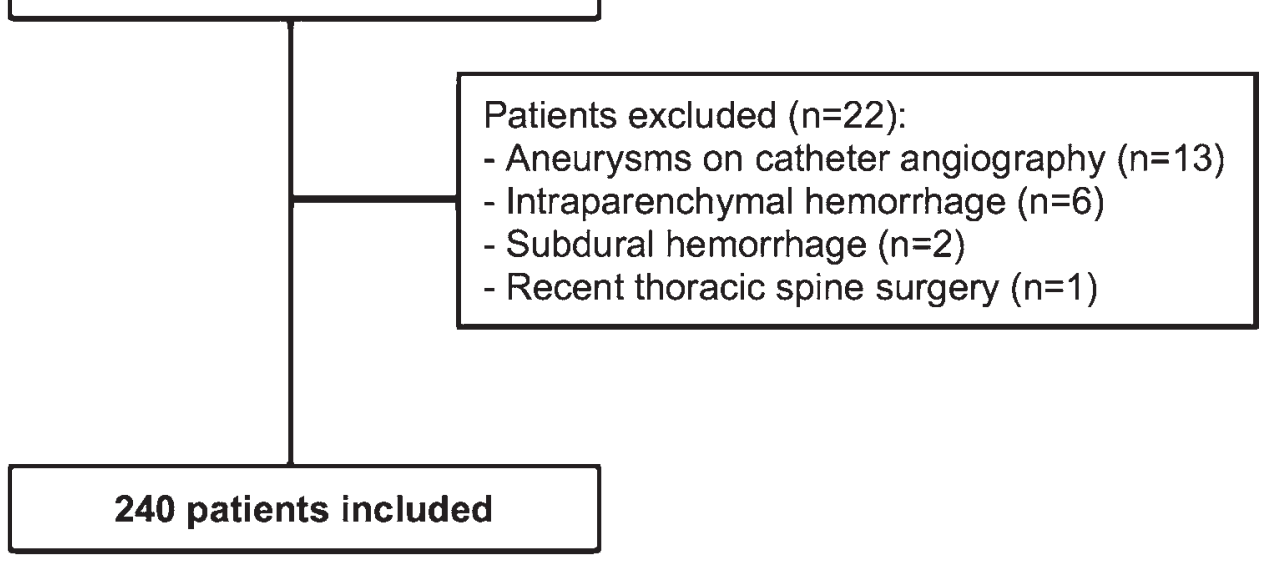

FIG. 1. Flow chart of the retrospective study. 
124 women, mean age 53 years, range $18-86$ years) were included in the analyses, with baseline characteristics as shown in Table 1. Hunt and Hess grades ranged from I to IV, with Grade I being the most common grade in our study population $(42 \%, 100 / 240)$. However, the Hunt and Hess grade was not recorded in the EMR in a similar number of our patients $(41 \%, 98 / 239)$.

Intracranial SAH was diagnosed with noncontrast head CT in 97\% (233/240) of patients (either obtained at our institution $[\mathrm{n}=209]$ or recorded in our EMR based on a study obtained at an outside hospital [ $\mathrm{n}=24])$. In $2.9 \%$ (7/240) of the patients, the head CT scan (either obtained at our institution $[n=5]$ or an outside hospital $[n=2]$ ) was negative for hemorrhage and the diagnosis was made by lumbar puncture. DSA was performed within 4 days of admission in all cases (median 12 hours, IQR 10 hours), and c-MRI was performed within 19 days of admission in all cases (median 24 hours, IQR 10 hours).

\section{Imaging Review}

Table 2 demonstrates the hemorrhage pattern and Fisher grades for included patients. The most common Fisher grade was 4, noted in 47\% (112/240) of cases. Review of cMRI showed a cause for the SAH in only $0.4 \%$ of patients $(1 / 240)$, which was a spinal vascular malformation initially diagnosed on CTA of the neck. In the 4 patients who underwent c-MRI without intravenous contrast, follow-up diagnostic imaging (DSA in 2 patients, and CTA head/ neck in 2 patients) did not reveal any spinal pathology, and the patients were discharged. In 3\% (7/240) of patients, an incidental finding was identified on cervical spine MR images, including a thyroid mass $(n=2)$, large perineural mass ( $\mathrm{n}=2$; both at the C7-T1 level), cervical syrinx ( $\mathrm{n}$ $=1)$, Chiari malformation Type I $(n=1)$, and nonspecific abnormal spinal cord signal $(\mathrm{n}=1)$, none of them explaining the cause of SAH.

\section{Meta-Analysis}

\section{Study Identification}

The initial search of the literature yielded 285 references. After exclusion of duplicate studies and studies that did not meet the inclusion criteria, 15 articles were reviewed in full text. Among these, 6 were eligible for inclusion. ${ }^{10,15}$, $16,19,22,25$ We added the results from our retrospective study to the meta-analysis data (Supplementary Fig. 1).

\section{Characteristics of Included Studies and Quality Assessment}

The characteristics of included studies are in Table 3. The number of patients with AN-SAH included in each study ranged from 68 to 240; and 470 of 897 patients (52\%) were male. Among studies that reported Hunt and Hess grades upon presentation, 97\% (852/876) were classified as a grade less than IV. Of the 897 patients with AN-SAH, 538 patients $(60 \%)$ underwent c-MRI and were included in this meta-analysis (Table 3).

Supplementary Table 1 summarizes the judgments of the methodological quality items for each included study according to the QUADAS-2 tool. ${ }^{23}$ The majority $(86 \%$ [6/7]) of studies had low risk of bias in patient selection, and all studies had low risk of bias in patient flow.
TABLE 1. Baseline characteristics for 240 patients with AN-SAH undergoing $\mathrm{C}-\mathrm{MRI}$ in a retrospective study

\begin{tabular}{cc}
\hline \multicolumn{1}{c}{ Variable } & $\%$ (no. of patients) \\
\hline Mean age \pm SD (yrs) & $53 \pm 13$ \\
\hline Sex & $48(116)$ \\
\hline Male & $52(124)$ \\
\hline Female & $54(130)$ \\
\hline Race & $26(61)$ \\
\hline White & $3(8)$ \\
\hline African American & $17(41)$ \\
\hline Others ${ }^{*}$ & \\
\hline Unknown & $41(98)$ \\
\hline Hunt \& Hess Grade & $42(100)$ \\
\hline Not reported & $2(5)$ \\
\hline I & $12(28)$ \\
\hline II & $4(9)$ \\
\hline III & $0(0)$ \\
\hline IV &
\end{tabular}

* Includes Hispanic, Asian, Native Hawaiian or other Pacific Islander, and multiple races.

However, given that the result of the c-MRI (in addition to cerebrovascular angiography, and CTA head and neck if available) was part of the reference standard to decide whether a patient had spinal pathology, the index test being evaluated (c-MRI) and the reference standard were not independent of each other, introducing risk of bias.

\section{Study Outcomes}

Data from c-MRI on 538 patients from 7 studies (including our current population) were pooled. Only 6 patients had positive c-MRI results, including 2 cervical ependymomas and 4 cervical vascular malformations. Figure 2 shows the forest plot of proportions and 95\% CIs for the individual studies. The pooled estimate for a positive c-MRI in a patient with AN-SAH was $1.3 \%(95 \% \mathrm{CI}$ $0.5 \%-2.5 \%$ ) with the fixed- and random-effects models. The between-study heterogeneity was not statistically significant $(\mathrm{Q}$ index 5.7, $\mathrm{p}=0.45)$. Because the studies were not found to be heterogeneous, the fixed-effects pooled estimate was used. There was no evidence of publication bias on the funnel plot (Supplementary Fig. 2).

TABLE 2. Fisher grade based on head CT for 240 patients with AN-SAH undergoing c-MRI in a retrospective study

\begin{tabular}{cc}
\hline Fisher Grade & $\%$ (no. of patients) \\
\hline No CT scan available & $14(34)$ \\
\hline 1 & $2(5)$ \\
\hline 2 & $7(17)$ \\
\hline 3 & $30(72)$ \\
\hline 4 & $47(112)$ \\
\hline
\end{tabular}


TABLE 3. Characteristics of included studies

\begin{tabular}{|c|c|c|c|c|c|c|c|}
\hline Study Characteristic & Rogg et al..$^{19}$ & Topcuoglu et al. ${ }^{22}$ & Little et al. ${ }^{16}$ & Lin et al. ${ }^{15}$ & Woodfield et al. ${ }^{25}$ & Germans et al. ${ }^{10}$ & Current Study \\
\hline Publication yr & 1999 & 2003 & 2007 & 2012 & 2014 & 2015 & 2017 \\
\hline No. of patients w/ AN-SAH & 71 & 86 & 100 & 68 & 242 & 90 & 240 \\
\hline Mean age (yrs) & NR & 54 & 48 & 59 & NR & 55 & 53 \\
\hline Males & $48(34)$ & $58(50)$ & $36(36)$ & $41(35)$ & $62(150)$ & $54(49)$ & $48(116)$ \\
\hline \multicolumn{8}{|l|}{ Hunt \& Hess grade } \\
\hline NR & $0(0)$ & $0(0)$ & $0(0)$ & $0(0)$ & $100(242)$ & $100(90)$ & $41(98)$ \\
\hline I & $79(56)$ & $72(62)$ & $13(17)$ & \multirow{3}{*}{$90(61)$} & - & - & $41(100)$ \\
\hline ॥ & $7(5)$ & $14(12)$ & $48(64)$ & & - & - & $2(5)$ \\
\hline III & $11(8)$ & $7(6)$ & $14(19)$ & & - & - & $12(28)$ \\
\hline IV \& V & $3(2)$ & $7(6)$ & $0(0)$ & $10(7)$ & - & - & $4(9)$ \\
\hline \multicolumn{8}{|l|}{ Fisher grade } \\
\hline NR & $72(51)$ & $0(0)$ & $25(25)$ & $0(0)$ & $1(2)$ & $57(51)$ & $14(34)$ \\
\hline 1 & $28(20)$ & $10(9)$ & $15(15)$ & $7(5)$ & $37(89)$ & $43(39)$ & $2(5)$ \\
\hline 2 & - & $69(59)$ & $17(17)$ & $34(23)$ & $17(42)$ & - & $7(17)$ \\
\hline 3 & - & $21(18)$ & $43(43)$ & \multirow{2}{*}{$59(40)$} & $23(56)$ & - & $30(72)$ \\
\hline 4 & - & $0(0)$ & $0(0)$ & & $22(53)$ & - & $47(112)$ \\
\hline No. of patients w/ c-MRI & $58(41)$ & $44(38)$ & $85(85)$ & $29(20)$ & $16(39)$ & $83(75)$ & $100(240)$ \\
\hline
\end{tabular}

$\mathrm{NR}=$ not reported.

All data given as the percentage (number) of patients, unless otherwise indicated.

\section{Discussion}

This retrospective study further supports the extremely low diagnostic yield of c-MRI in the evaluation of AN$\mathrm{SAH}$. There was a diagnostic yield of $0.4 \%$ for c-MRI when evaluating patients who underwent our standardized institutional treatment protocol of DSA and head CTA followed by brain and cervical spine MRI. Furthermore, our meta-analysis of 538 patients with AN-SAH produced a weighted pooled estimate of $1.3 \%$ (95\% CI $0.5 \%-2.5 \%)$ for the diagnostic yield of c-MRI.

The use of c-MRI in the AN-SAH population was initially recommended in a few case reports of patients with AN-SAH in whom a spinal pathology was found on cervical spine MR images., This recommendation was fur- ther emphasized by a study published in 1999, reporting 1 case of a spinal vascular malformation in 41 patients with AN-SAH who underwent c-MRI. ${ }^{19}$ However, more recent studies have demonstrated that the likelihood of finding a spinal source for AN-SAH in this patient population is extremely low (between $0 \%$ and $4 \%$ ) and have questioned the use of c-MRI: ${ }^{10,22}$ specifically, in patients with a perimesencephalic pattern of hemorrhage, all of the reported anecdotal cases of vascular etiologies were intracranial. ${ }^{24,26}$ Studies focusing on MRI of the craniocervical junction ${ }^{17}$ yielded similar results but were not included in our metaanalysis, as a dedicated cervical spine MR image was not obtained. Other studies have recommended the use of cMRI only in selected cases in which there are signs and

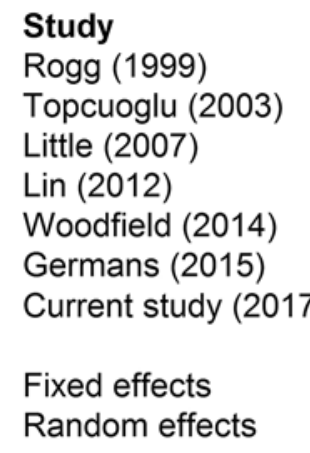

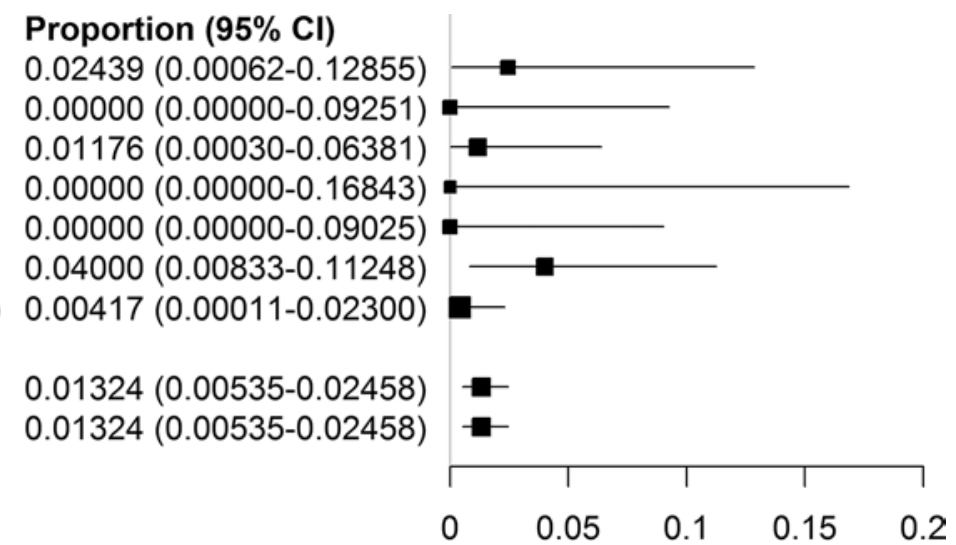

FIG. 2. Forest plot of proportions and $95 \%$ Cls according to the study. Included are statistics for the fixed-effects and random-effects models. The sizes of boxes indicating proportion estimates are weighted by the sample size in each study, except the boxes for fixed- and random-effects estimates. 
symptoms raising the suspicion for spinal pathology, ${ }^{16,25}$ or for patients with a second clinical episode. ${ }^{27}$ However, all of these studies have been limited by small sample sizes.

It is important to identify spinal etiologies for intracranial SAH, such as intradural vascular lesions and neoplasms, to prevent recurrent hemorrhage that may result in a catastrophic outcome. ${ }^{25}$ However, given the low diagnostic yield of c-MRI, its cost-effectiveness is questionable and beyond the scope of the current study. Using simplified methodology, our meta-analysis of the literature demonstrated that the weighted pooled estimate diagnostic yield was $1.3 \%$. Based on a prior cost-effectiveness analysis, ${ }^{21}$ untreated SAH is estimated to cost $\$ 42,000-\$ 82,000$ per patient, with an average of $\$ 59,000$ (calibrated to 2003 US dollars), regardless of the payer, with the final cost dependent upon the patient's characteristics and origin of SAH. The Medicare global allowable fee for c-MRI in 2003 was approximately $\$ 1000$ (https://www.cms.gov/apps/ physician-fee-schedule/). Restricting the payer to Medicare (which likely underestimates the total cost), if 1000 patients undergo a c-MRI for evaluation of AN-SAH, the estimated cost is $\$ 1,000,000$. Based upon our metaanalysis diagnostic yield of c-MRI of $1.3 \%$, if 13 patients with a potentially treatable cause of SAH go untreated (with an average cost of $\$ 59,000$ ), there is an estimated cost of $\$ 767,000$, which is less than performing a screening c-MRI for all patients. We therefore propose that the addition of c-MRI in the routine evaluation of AN-SAH to be highly unlikely to contribute diagnostic benefit in such patients and is not cost-effective. We understand that final reimbursement of c-MRI will vary by insurance type, as will the cost of not treating $\mathrm{SAH}$ (which may also require factoring for life-years lost due to morbidity and mortality), and, therefore, the cost estimates presented here are grossly simplified. Our findings suggest that forgoing unnecessary c-MRI in the diagnostic workup of AN-SAH may mitigate a portion of health care cost and overutilization, although rigorous cost-benefit analysis is lacking at this time and the focus of future research.

Medicolegal aspects are often a common reason for referring physicians to order c-MRI for patients with ANSAH. A prior study of neurosurgical negligence claims in the US has shown that failed diagnosis of vascular etiology has been common after spinal surgery, intracranial surgery, and trauma. ${ }^{7}$ However, it is not clear how many of these cases resulted from a cervical spine etiology in the setting of AN-SAH. A similar study in the United Kingdom from 2002 to 2012, limited to spinal vascular etiologies, reported only 3 cases (1.2\% of total cases), and only 2 of them were successfully closed; again, it is not clear whether they were in an AN-SAH setting. ${ }^{11}$ Regardless, we believe this should not be a barrier for appropriate utilization of health resources. If there are no clinical/neurological findings to suggest spinal origin and the patient has a negative head and neck CTA and DSA, we argue that ordering c-MRI solely on a medicolegal basis is not the best practice, as the likelihood of identifying a cause is less than $1 \%$.

The present study contributes to the previously relatively sparse volume of data examining the use of c-MRI in intracranial $\mathrm{SAH}$, where a limited number of relatively small studies (including 35-75 patients), each with disparate methodologies, may leave definitive conclusions unresolved..$^{10,15,16,19,22,25}$ One of the advantages of the current retrospective study over currently available studies is the larger sample size, which provides sufficient power $(80 \%)$ to statistically assess diagnostic yield of c-MRI. With 240 patients in this study, the $95 \%$ estimated CI around a point estimate proportion of 0.015 (the weighted average of proportions from the existing 6 studies) is 0.004 to 0.040 , which equals a CI width of 0.036 . The estimated CI width for a study with 50 patients is 0.098 , nearly 3 times larger.

\section{Limitations of the Study}

We acknowledge several study limitations, including those inherent to our retrospective study design. These results were drawn almost entirely from a large, singleinstitution registry of patients, with established practice patterns, which may serve to mitigate some heterogeneity or nonuniformity across the data. For example, the majority of patients in our study presented with a Fisher Grade 3 or $4 \mathrm{SAH}$, which might be related to our institution being a tertiary academic referral center. Furthermore, due to the same reason, several patients were transferred from outside facilities, and, therefore, their preceding workup prior to transfer may have varied by institution. To the extent possible, we attempted to obtain all relevant information for those patients from the EMR, and to exclude any patients for whom a portion of their workup would lead to their exclusion. In $2 \%$ of included patients in the current retrospective study, the c-MRI was performed without intravenous contrast administration, which may slightly limit the evaluation of spinal etiologies. However, one of these patients underwent a contrast-enhanced neck MR angiogram, allowing limited evaluation of the spine, and follow-up imaging in all these patients did not reveal any spinal pathology. The presence of only 1 positive case on cervical spine MR images for the etiology of SAH prohibited a subgroup analysis based on pattern or severity of hemorrhage, age, sex, or other risk factors. Additionally, the meta-analysis was limited in performing a subgroup analysis based on pattern of hemorrhage, due to the small number of positive cervical spine MR images and because the pattern of hemorrhage for individual patients was not reported in the prior studies. Finally, our study is limited in determining whether c-MRI might have a higher diagnostic yield in patients with a second episode of SAH, as it was not clear what percentage of patients included in the current meta-analysis had a second episode of SAH.

\section{Conclusions}

Cervical spine MRI following AN-SAH, in the absence of clinical/neurological findings to suggest a spinal etiology, has a very low diagnostic yield. As such, routine MRI for possible cervical sources of intracranial AN-SAH is not evidence based.

\section{Acknowledgments}

Research reported in this publication was supported in part by the Biostatistics and Bioinformatics Shared Resource of the Winship Cancer Institute of Emory University and the NIH/NCI 
(grant no. P30CA138292). The content is solely the responsibility of the authors and does not necessarily represent the official views of the NIH.

\section{References}

1. Andaluz N, Zuccarello M: Yield of further diagnostic workup of cryptogenic subarachnoid hemorrhage based on bleeding patterns on computed tomographic scans. Neurosurgery 62:1040-1047, 2008

2. Canneti B, Mosqueira AJ, Nombela F, Gilo F, Vivancos J: Spontaneous subarachnoid hemorrhage with negative angiography managed in a stroke unit: clinical and prognostic characteristics. J Stroke Cerebrovasc Dis 24:2484-2490, 2015

3. Chalif DJ, Black K, Rosenstein D: Intradural spinal cord tumor presenting as a subarachnoid hemorrhage: magnetic resonance imaging diagnosis. Neurosurgery 27:631-634, 1990

4. Corriero G, Iacopino DG, Valentini S, Lanza PL: Cervical neuroma presenting as a subarachnoid hemorrhage: case report. Neurosurgery 39:1046-1049, 1996

5. de Rooij NK, Linn FH, van der Plas JA, Algra A, Rinkel GJ: Incidence of subarachnoid haemorrhage: a systematic review with emphasis on region, age, gender and time trends. J Neurol Neurosurg Psychiatry 78:1365-1372, 2007

6. DerSimonian R, Laird N: Meta-analysis in clinical trials. Control Clin Trials 7:177-188, 1986

7. Fager CA: Malpractice issues in neurological surgery. Surg Neurol 65:416-421, 2006

8. Fisher CM, Kistler JP, Davis JM: Relation of cerebral vasospasm to subarachnoid hemorrhage visualized by computerized tomographic scanning. Neurosurgery 6:1-9, 1980

9. Freeman MF, Tukey JW: Transformations related to the angular and the square root. Ann Math Stat 21:607-611, 1950

10. Germans MR, Coert BA, Majoie CB, van den Berg R, Lycklama À Nijeholt G, Rinkel GJ, et al: Yield of spinal imaging in nonaneurysmal, nonperimesencephalic subarachnoid hemorrhage. Neurology 84:1337-1340, 2015

11. Hamdan A, Strachan RD, Nath F, Coulter IC: Counting the cost of negligence in neurosurgery: Lessons to be learned from 10 years of claims in the NHS. Br J Neurosurg 29:169-177, 2015

12. Higgins JPT, Green S (eds): Cochrane Handbook for Systematic Reviews of Interventions, Version 5.1.0. London: The Cochrane Collaboration, 2011 (http://handbook. cochrane.org) [Accessed June 6, 2017]

13. Higgins JPT, Thompson SG: Quantifying heterogeneity in a meta-analysis. Stat Med 21:1539-1558, 2002

14. Hunt WE, Hess RM: Surgical risk as related to time of intervention in the repair of intracranial aneurysms. J Neurosurg 28:14-20, 1968

15. Lin N, Zenonos G, Kim AH, Nalbach SV, Du R, Frerichs $\mathrm{KU}$, et al: Angiogram-negative subarachnoid hemorrhage: relationship between bleeding pattern and clinical outcome. Neurocrit Care 16:389-398, 2012

16. Little AS, Garrett M, Germain R, Farhataziz N, Albuquerque FC, McDougall CG, et al: Evaluation of patients with spontaneous subarachnoid hemorrhage and negative angiography. Neurosurgery 61:1139-1151, 2007

17. Maslehaty H, Petridis AK, Barth H, Mehdorn HM: Diagnostic value of magnetic resonance imaging in perimesencephalic and nonperimesencephalic subarachnoid hemorrhage of unknown origin. J Neurosurg 114:1003-1007, 2011

18. Moher D, Liberati A, Tetzlaff J, Altman DG: Preferred reporting items for systematic reviews and meta-analyses: the PRISMA statement. Ann Intern Med 151:264-269, W64, 2009

19. Rogg JM, Smeaton S, Doberstein C, Goldstein JH, Tung GA, Haas RA: Assessment of the value of MR imaging for exam- ining patients with angiographically negative subarachnoid hemorrhage. AJR Am J Roentgenol 172:201-206, 1999

20. Sterne JA, Egger M: Funnel plots for detecting bias in metaanalysis: guidelines on choice of axis. J Clin Epidemiol 54:1046-1055, 2001

21. Takao H, Nojo T: Treatment of unruptured intracranial aneurysms: decision and cost-effectiveness analysis. Radiology 244:755-766, 2007

22. Topcuoglu MA, Ogilvy CS, Carter BS, Buonanno FS, Koroshetz WJ, Singhal AB: Subarachnoid hemorrhage without evident cause on initial angiography studies: diagnostic yield of subsequent angiography and other neuroimaging tests. J Neurosurg 98:1235-1240, 2003

23. Whiting PF, Rutjes AW, Westwood ME, Mallett S, Deeks JJ, Reitsma JB, et al: QUADAS-2: a revised tool for the quality assessment of diagnostic accuracy studies. Ann Intern Med 155:529-536, 2011

24. Wijdicks EF, Schievink WI: Perimesencephalic nonaneurysmal subarachnoid hemorrhage: first hint of a cause? Neurology 49:634-636, 1997

25. Woodfield J, Rane N, Cudlip S, Byrne JV: Value of delayed MRI in angiogram-negative subarachnoid haemorrhage. Clin Radiol 69:350-356, 2014

26. Yaghi S, Oomman S, Keyrouz SG: Non-aneurysmal perimesencephalic subarachnoid hemorrhage caused by a cavernous angioma. Neurocrit Care 14:84-85, 2011

27. Yap L, Dyde RA, Hodgson TJ, Patel UJ, Coley SC: Spontaneous subarachnoid hemorrhage and negative initial vascular imaging-should further investigation depend upon the pattern of hemorrhage on the presenting CT? Acta Neurochir (Wien) 157:1477-1484, 2015

\section{Disclosures}

The authors report no conflict of interest concerning the materials or methods used in this study or the findings specified in this paper.

\section{Author Contributions}

Conception and design: Sadigh, Holder, Allen. Acquisition of data: Sadigh, Holder, Dehkharghani, Allen. Analysis and interpretation of data: Sadigh, Switchenko, Dehkharghani, Allen. Drafting the article: Sadigh. Critically revising the article: all authors. Reviewed submitted version of manuscript: all authors. Approved the final version of the manuscript on behalf of all authors: Sadigh. Statistical analysis: Sadigh, Switchenko. Administrative/ technical/material support: Sadigh. Study supervision: Allen.

\section{Supplemental Information \\ Online-Only Content}

Supplemental material is available with the online version of the article.

Supplementary Figures and Table. https://thejns.org/doi/suppl/ 10.3171/2017.4.JNS163114.

\section{Previous Presentations}

The abstract of this paper was presented at the 2015 Radiological Society of North America Annual Meeting, November 29 to December 4, in Chicago, Illinois.

\section{Correspondence}

Gelareh Sadigh, Department of Radiology and Imaging Sciences, Emory University School of Medicine, 1364 Clifton Rd. NE, Atlanta, GA 30322. email: gsadigh@emory.edu. 